\title{
Evaluation of Survival and Treatment Toxicity With High-Dose-Rate Brachytherapy With Cobalt 60 in Carcinoma of Cervix
}

\author{
Afshin Rakhsha, ${ }^{1}$ Amir Shahram Yousefi Kashi, ${ }^{1, *}$ and Seied Mohsen Hoseini ${ }^{2}$ \\ ${ }_{1}^{1}$ Department of Radiation Oncology, Shohada-e-Tajrish Hospital, Faculty of Medicine, Shahid Beheshti University of Medical Sciences, Tehran, IR Iran \\ ${ }^{2}$ Department of Radiation Oncology, Urmia University of Medical Sciences, Urmia, IR Iran \\ ${ }^{*}$ Corresponding author: Amir Shahram Yousefi Kashi, Department of Radiation Oncology, Shohada-e-Tajrish Hospital, Faculty of Medicine, Shahid Beheshti University of Medical \\ Sciences, Tehran, IR Iran. Tel/Fax: +98-2122739200, E-mail: shahpoo2002@yahoo.com
}

Received: July 20, 2015; Accepted: July 21, 2015

Background: Cervical cancer remains to be a major health problem and cancer-related cause of death among women in developing countries such as Iran where the most cases are diagnosed in locally advanced stage.

Objectives: This cross sectional-analytic study aims to report outcome 154 patients with carcinoma of cervix were treated with external beam radiation therapy (EBRT) and high-dose-rate (HDR) brachytherapy with cobalt 60 (Co-60) remote after loading system.

Patients and Methods: A total of 154 patients with the international federation of gynecologist and oncologist (FIGO) stages I-IVA with histopathologically confirmed carcinoma of cervix, followed by the radiation-oncology ward of Shohada-e-Tajrish Hospital in Tehran, Iran, between February 2008 and March 2015. They were completed their scheduled EBRT and HDR brachytherapy with Co-60 remote after loading system. Out of this, 132 patients completed their standard follow up protocol. They were analyzed for 3-year disease-free survival (DFS), 3-year overall survival (OS) incidence of acute and late complications for HDR brachytherapy.

Results: Fourteen patients (9.1\%) were in stage I(FIGO classification), 8 (5.2\%) were in stage IIA, 26 (16.9\%) were in stage IIB, 100 (64.9\%) were in stage III, and 6 (3.9\%) were in stage IVA. The follow up duration was between 6 - 60 months with a median of 38 months. Overall rectal and bladder treatment toxicity rates were $33.7 \%$. The 3-year DFS rate was $85.7 \%, 70.7 \%, 41 \%$ and $16.6 \%$ for stages I, II, III, IVA respectively. Favorable prognostic factors in univariate and multivariate analysis were early stage, tumor size $<4 \mathrm{~cm}$ (after adjusting for the residual disease after radiation), no pelvic lymph node involvement and 1 week Gap between EBRT and HDR brachytherapy in 3-year DFS $(\mathrm{P}=0.001, \mathrm{P}=0.012, \mathrm{P}=$ $0.005, \mathrm{P}=0.005$, respectively). The 3 -year OS rate was $85.7 \%, 76.4 \%, 42 \%$, and $33.3 \%$ for stages I, II, III, and IVA, respectively. Favorable prognostic factors in univariate and multivariate analysis were early stage, tumor size $<4 \mathrm{~cm}$, no pelvic lymph node involvement, 1 week gap between EBRT and HDR brachytherapy and no distant metastasis (during the follow up) in 3-year OS ( $\mathrm{P}=0.001, \mathrm{P}=0.002, \mathrm{P}=0.002, \mathrm{P}=0.002, \mathrm{P}=$ 0.001, respectively).

Conclusions: HDR brachytherapy with Co-60 remote after loading system was successful and it showed HDR brachytherapy in treating patients with carcinoma of cervix was effective after EBRT with acceptable rectal and bladder complications.

Keywords: Brachytherapy; Disease Free Survival; Overall Survival; Treatment

\section{Background}

Cervical cancer remains to be a major health problem and cancer-related cause of death among women in developing countries such as Iran where the most cases are diagnosed in locally advanced stage (1).

There are different techniques radiotherapy with were used for the carcinoma of uterine cervix. Several studies have confirmed that local control rates, disease-free survival (DFS) and overall survival (OS) are significantly improved with the combination of external beam radiation therapy (EBRT) and intracavitary radiotherapy (ICRT) $(2,3)$.

ICRT is the main treatment protocol of radiation therapy for the patients with cervical cancer and is always delivered after EBRT for radical treatment. ICRT can deliver a huge proportional radiation dose to the microscopic or macroscopic residual tumor after EBRT while sparing the adjacent normal organs such as bladder and rectum (4).
There are known three methods for intracavitary brachytherapy according to ICRU report 38:

- Low-dose rate (LDR) brachytherapy with dose rate between 0.4 and 2 Gy/h. LDR brachytherapy can be delivered by manual or remote afterloading systems.

- Medium-dose rate (MDR) brachytherapy with dose rate between $2 \mathrm{~Gy} / \mathrm{h}$ and $12 \mathrm{~Gy} / \mathrm{h}$. MDR brachytherapy can also be used by manual or remote afterloading systems.

- High-dose rate (HDR) brachytherapy, and just remote afterloading systems must be used due to the very high source radioactivity (5).

There are some disadvantages of LDR brachytherapy and MDR brachytherapy than the HDR brachytherapy such as unnecessary radiation exposure of the radiation oncologist and other staffs, prolonged treatment time that may lead to thromboembolism events, and the risk of movement of the applicators, but HDR brachytherapy

Copyright (C) 2015, Iranian Journal of Cancer Prevention. This is an open-access article distributed under the terms of the Creative Commons Attribution-NonCommercial 4.0 International License (http://creativecommons.org/licenses/by-nc/4.0/) which permits copy and redistribute the material just in noncommercial usages, provided the original work is properly cited. 
is an outpatient treatment with had a more convenient modality for cervical cancer patients $(6,7)$.

Traditionally, High Dose Rate afterloaders have been based on Iridium 192 or cobalt 60 (8).

\section{Objectives}

The present study reviewed our experience with HDR brachytherapy with cobalt 60 (Co-60) remote after loading system to evaluate treatment outcome treated cervical cancer patients between 2008 and 2015 after EBRT, to identify prognostic factors for 3-year OS, 3-year DFS and incidence of acute and late gastrointestinal and genitourinary complications.

\section{Patients and Methods}

This was a cross sectional-analytic study that data obtained from patient medical records. Staging of cancer cervix was performed based on the international federation of gynecology and obstetrics (FIGO) criteria in our center or other centers.

A total of 154 patients who diagnosed with histopathologically confirmed carcinoma of cervix, followed by the radiation-oncology ward of Shohada-e-Tajrish hospital in Tehran, Iran between February 2008 and March 2015. They were completed their scheduled EBRT + HDR ICRT with Co-60 remote after loading system treatment radiotherapy. They were analyzed for 3-year DFS (residual disease, local recurrence, and distant metastases), 3-year OS and incidence of acute and late treatment complications for HDR-ICRT.

All the patients were treated initially with external beam irradiation in our center or other centers with total dose 45 Gy-54 Gy with or without concomitant chemotherapy. After completion of EBRT all Patients were planned for brachytherapy within 1 to 3 weeks and more due to the great number of patients with three to five fractions of HDR brachytherapy for each patient with a week's interval.

We used Co-60 HDR after-loading system for intracavitary radiotherapy with one tandem and two ovoid applicators based on the Manchester System. There were three different sizes of tandems and ovoids based on the length of uterus and expansion of the vaginal wall. We performed meticulous vaginal packing with betadine gauze packs to get away the bladder and rectum from the applicators. In brachytherapy room, tumor regression was evaluated and endocervical canal was dilated by a radiation oncologist with assistance of two nurses.

All the tandem and ovoids insertions, X-ray images, and treatments were done in a standard brachytherapy room with X-ray equipment by a radiation oncologist and two well trained nurses.

Treatment planning was done by a radiation oncologist and a medical radiation physicist for the treatment planning system.

The brachytherapy dose was calculated based on EQD
$2 \mathrm{~Gy}+\mathrm{EQD}$ HDR = Total dose to point A (80 - 90 Gy) and delivered to the point $A$. The treatment overall time was between 20 to 40 minutes.

After the completion of treatment, all 154 patients were followed by the radiation-oncology ward of Shohada-eTajrish hospital between February 2008 and March 2015 with standard guide lines every 3 months for the first 2 years, every 6 months for the next 3 years and annually afterward.

During each follow up, clinical examination was done by a radiation oncologist in each appointment and cervical cytology was taken by a gynecologic oncologist at the first follow-up and annually afterward. Paraclinic examination such as blood test, X-ray, ultrasonography, bone scan, CT scan and MRI were used based on the patients complains and suspected examination findings to rule out any locoregional and distant metastasis. All local recurrences disease was histologically confirmed. Recurrences were defined as distant if they occurred in the Para-aortic lymph nodes or outside the pelvis. Patients with rectal or bladder complications were evaluated by the common toxicity criteria by radiation therapy oncology group (RTOG). Patients considered lost to follow up who did not visit after any appointments. 22 patients were excluded from the study because of they did not complete our standard follow up protocol.

Patients without any locoregional recurrence or distant metastases until the last follow up were considered as disease free. Follow-up time was defined by the interval between the last day of HDR ICRT and the last information about the patient. The follow up duration was between 6 - 60 months. 3-year DFS rates were calculated from date of the last day of HDR ICRT until the date of first recurrence or progression or distant metastases. 3-year OS rates were calculated from date of the last day of HDR ICRT until the date of death.

The ethical regulations dictated in the act provided by Shahid Beheshti University of Medical Sciences (reference number of research ethics committee: 400) were strictly observed. The data were strictly preserved without using the patient's names.

The examination of three-year DFS and the three-year OS by univariable analyses was performed by log rank test. For multivariate analysis, the Cox proportional hazards model was used to assess the strengths of various histoclinical and treatment factors with three-year DFS and the three-year OS. The statistical analyses were performed by using software SPSS version 21 (SPSS IBM).

\section{Results}

154 patients were the subjects for our study. The follow up duration was between 6 - 60 months with a median of 38 months. Their median age was 57.4 years (range, 33 to 86 years). Fourteen patients ( $9.1 \%$ ) were in stage I (FIGO classification), 8 (5.2\%) were in stage IIA, 26 (16.9\%) were in stage IIB, 100 (64.9\%) were in stage III, and 6 (3.9\%) were in stage IVA. 102 patients (66.2\%) had tumor size $\geq 4 \mathrm{~cm}$. 
Rakhsha A et al.

137 patients (89\%) had squamous cell carcinoma and 13 patients $(8.4 \%)$ had adenocarcinoma in their final histology reports. 132 patients $(85.7 \%)$ received concomitant chemotherapy with EBRT. 19 patients (12.3\%) was detected during the follow up with distant metastasis, which consistent of lung 6, bone 4, liver 3, Para-aortic lymph node 6, Supraclavicular lymph node and Para-aortic lymph node 2. 44 patients (28.6\%) were diagnosed with pelvic lymph node involvement based on imaging studies. The median total dose of EBRT was $50 \mathrm{~Gy}$ (range, 45 to $54 \mathrm{~Gy}$ ), median total dose of BT was $20 \mathrm{~Gy}$ (range, 16 to $24 \mathrm{~Gy}$ ) and median total dose to the point A was $80 \mathrm{~Gy}$ (range, 70 to $90 \mathrm{~Gy}$ ). The mean fractions number of BT was 4 fractions (range, 3 to 5 fractions) and the median gap between EBRT and HDR ICRT was 2 weeks (range, 1 to 5 weeks). Table 1 shows patient, tumor characteristics and treatment factors of 154 adult patients included in this study.

Acute and late gastrointestinal (rectal) and genitourinary (bladder) complications based on RTOG criteria were seen $6.5 \%, 3.9 \%$ and $16.2 \%$, 7.1\% respectively. Overall rectal and bladder complications were seen in 16 (10.4\%) and 36 (23.3\%) patients. No patients died of any irradiated-related causes (grade 5).

Four patients experienced grade 1 and grade 2 acute radiation proctitis and six patients had grade 3 acute proctitis. One patient had grade 1 and grade 2 late radiation proctitis and six experienced grade 3 late proctitis. There was no patient in grade 4 acute and chronic radiation proctitis. Twenty patients experienced grade 1 and 2 acute radiation cystitis, four patients experienced grade 3 acute cystitis and one patient had grade 4 acute cystitis.

Two patients experienced grade 1 and grade 2 late genitourinary toxicities, eight patients experienced grade 3 late genitourinary toxicities and one patient had grade 4. The most common acute and late gastrointestinal (rectal) and genitourinary (bladder) toxicities were in stage III patients. Table 2 shows acute and late toxicities based on RTOG criteria and stage of tumor in patients.

Overall, $22(14.28 \%)$ patients were excluded because of lost to follow up after completion of EBRT and HDR ICRT (1patient in stage I, 5 patients in stage II, 14 patients in stage III and 2 patients In stage IVA) and 78 (50.64\%) patients were disease free (12 patients were in stage I, 24 patients were in stage II, 41 patients were in stage III and only 1 patient was in stage IVA). The median follow-up period was 38 months overall, and 36 months for patients who did not die of their cervical cancer.

The 3 year DFS rate was $85.7 \%, 70.7 \%, 41 \%$ and $16.6 \%$ for stages I, II, III, IVA respectively.

According to Kaplan-Meier 3-year DFS analysis and Logrank test in univariate analysis and Cox regression model in multivariate analysis, four factors were statistically significant relation between 3-year DFS and early stage ( $P$ $=0.001$, Odd Ratio $(\mathrm{OR})=2.31,95 \% \mathrm{CI}=1.13-4.52$ ), tumor size $\geq 4 \mathrm{~cm}$ (after adjusting for the residual disease after radiation $)(\mathrm{P}=0.012, \mathrm{OR}=2.19,95 \% \mathrm{CI}=1.01-4.40)$, pelvic lymph node involvement $(\mathrm{P}=0.005, \mathrm{OR}=2.25,95 \% \mathrm{CI}=$
1.07 - 4.46) and 1 week Gap between EBRT and HDR ICRT (P $=0.005, \mathrm{OR}=1.74,95 \% \mathrm{CI}=0.99-2.96)$ (Table 3$)$. Then, the favorable prognostic factors in our study in univariate and multivariate analysis were: early stage, tumor size $<$ $4 \mathrm{~cm}$, no pelvic lymph node involvement and 1 week Gap between EBRT and HDR ICRT. There was no statistically significant relationship between 3-year DFS and histology tumor (after adjusting for the residual disease after radiation) and mean total dose to the point $\mathrm{A}$ by univariate and multivariate analysis (Table 3 ).

Table 1. Patient, Tumor Characteristics and Treatment Factors of 154 Adult Patients With Cervical Caicinoma ${ }^{\text {a,b }}$

\begin{tabular}{lc}
\hline Factors & Total $(\mathbf{n}=\mathbf{1 5 4})$ \\
\hline Age, $\mathbf{y}$ & $57.4(33-86)$ \\
FIGO Stage & \\
\hline I & $14(9.1)$ \\
IIA & $8(5.2)$ \\
IIB & $26(16.9)$ \\
III & $100(64.9)$ \\
IVA & $6(3.9)$
\end{tabular}

\begin{tabular}{|c|c|}
\hline \multicolumn{2}{|l|}{ Tumor Size, cm } \\
\hline Tumor size $\geq 4$ & $102(66.2)$ \\
\hline Tumor size $<4$ & $52(33.8)$ \\
\hline
\end{tabular}

\section{Histology Type}

Squamous cell carcinoma $\quad 137(89)$

Adenocarcinoma $13(8.4)$

Adenosquamous carcinoma $\quad 4(2.6)$

Lymph node enlargement

\begin{tabular}{lc}
\hline None & $102(66.2)$ \\
\hline Pelvic alone & $44(28.6)$ \\
\hline Pelvic and para-aortic & $8(5.2)$ \\
\hline Mean dose of EBRT, Gy & $50(45-54)$ \\
\hline Concomitant chemotherapy with EBRT & \\
\hline Yes & $132(85.7)$ \\
\hline No & $22(14.3)$ \\
\hline Mean total dose of BT, Gy & $20(16-24)$ \\
\hline Mean total dose to the point A, Gy & $80(70-90)$ \\
\hline Mean of number fractions of BT & $4(3-5)$ \\
Gap between EBRT and HDR ICRT, wk & $2(1-3)$ \\
\hline Follow up (For all 154 patients) & $38(6-60)$ \\
\hline
\end{tabular}

a Abbreviations: BT, brachytherapy; EBRT, external beam radiotherapy; FIGO, international federation of gynecology and obstetrics; HDR ICRT, high dose rate intracavitary radiotherapy.

b Data are presented as median (range) or No. (\%). 
Rakhsha A et al.

\begin{tabular}{|c|c|c|c|c|c|c|c|c|c|c|}
\hline & \multicolumn{5}{|c|}{ Acute } & \multicolumn{5}{|c|}{ Late } \\
\hline & Go & G1 and G2 & G3 & G4 & Total & Go & G1 and G2 & G3 & G4 & Total \\
\hline \multicolumn{11}{|c|}{ Gastrointestinal Complications Stage } \\
\hline I & 14 & 0 & 0 & 0 & $14(9.1)$ & 14 & 0 & 0 & 0 & $14(9.1)$ \\
\hline IIA & 7 & 1 & 0 & 0 & $8(5.2)$ & 8 & 0 & 0 & 0 & $8(5.2)$ \\
\hline IIB & 24 & 1 & 1 & 0 & $26(16.9)$ & 24 & 0 & 2 & 0 & $26(16.9)$ \\
\hline III & 94 & 2 & 4 & 0 & $100(64.9)$ & 96 & 1 & 3 & 0 & $100(64.9)$ \\
\hline IVA & 5 & 0 & 1 & 0 & $6(3.9)$ & 6 & 0 & 0 & 0 & $6(3.9)$ \\
\hline Total & 144 & 4 & 6 & 0 & $154(100)$ & 148 & 1 & 5 & 0 & $154(100)$ \\
\hline \multicolumn{11}{|c|}{ Genitourinary Complications Stage } \\
\hline I & 14 & 0 & 0 & 0 & $14(9.1)$ & 14 & 0 & 0 & 0 & $14(9.1)$ \\
\hline IIA & 7 & 1 & 0 & 0 & $8(5.2)$ & 8 & 0 & 0 & 0 & $8(5.2)$ \\
\hline IIB & 24 & 4 & 1 & 0 & $26(16.9)$ & 24 & 0 & 2 & 0 & $26(16.9)$ \\
\hline III & 94 & 14 & 3 & 1 & $100(64.9)$ & 92 & 2 & 5 & 1 & $100(64.9)$ \\
\hline IVA & 5 & 1 & 0 & 0 & $6(3.9)$ & 5 & 0 & 1 & 0 & $6(3.9)$ \\
\hline Total & 129 & 20 & 4 & 1 & $154(100)$ & 143 & 2 & 8 & 1 & $154(100)$ \\
\hline
\end{tabular}

a Abbreviation: G: grade.

Table 3. Three-year Disease Free Mean Survival Rate According to Univariate and Multivariate Analysis (After Adjusting for the Residual Disease After Radiation for the Histology and Tumor Size ${ }^{\text {a }}$

\begin{tabular}{|c|c|c|c|c|c|c|}
\hline Factor & $\begin{array}{c}\text { No. of } \\
\text { Patients }\end{array}$ & $\begin{array}{l}\text { Three-Year } \\
\text { DFS }\end{array}$ & $\begin{array}{l}\text { Univariate Analysis } \\
\text { P Value }\end{array}$ & $\begin{array}{c}\text { Odd } \\
\text { Ratio }\end{array}$ & 95\% CI & $\begin{array}{c}\text { Multivariate } \\
\text { Analysis P Value }\end{array}$ \\
\hline Stage & & & 0.03 & 2.31 & $1.13-4.52$ & 0.001 \\
\hline I + IIA + IIB (early stage) & 48 & $36(75)$ & & & & \\
\hline III + IVA (locally advanced stage) & 106 & $42(39.62)$ & & & & \\
\hline Histology (after adjusting) & & & 0.11 & 1.52 & $0.68-3.49$ & 0.32 \\
\hline Squamous cell carcinoma & 137 & $65(60.58)$ & & & & \\
\hline Adenocarcinoma & 13 & $6(69.23)$ & & & & \\
\hline Adenosquamous carcinoma & 4 & $2(50)$ & & & & \\
\hline Tumor size (after adjusting), cm & & & 0.04 & 2.19 & $1.01-4.40$ & 0.005 \\
\hline$\geq 4$ & 102 & $37(52.85)$ & & & & \\
\hline$<4$ & 52 & $34(77.27)$ & & & & \\
\hline Pelvic lymph node enlargement & & & 0.03 & 2.25 & $1.07-4.46$ & 0.005 \\
\hline No & 102 & $66(64.7)$ & & & & \\
\hline Yes & 52 & $15(28.8)$ & & & & \\
\hline Gap between EBRT and HDR ICRT, wk & & & 0.009 & 1.74 & $0.99-2.96$ & 0.005 \\
\hline 1 & 95 & $69(72.63)$ & & & & \\
\hline$\geq 3$ & 59 & $29(49.15)$ & & & & \\
\hline Mean total dose to the point $\mathrm{A}, \mathrm{Gy}$ & & & 0.082 & 1.18 & $0.79-1.75$ & 0.099 \\
\hline$<80$ & 80 & $44(55)$ & & & & \\
\hline$\geq 80$ & 74 & $31(41.9)$ & & & & \\
\hline
\end{tabular}

a Abbreviations: DFS, disease free mean survival; EBRT, external beam radiotherapy; HDR ICRT, high dose rate intracavitary radiotherapy.

The 3 year OS rate was $85.7 \%, 76.4 \%, 42 \%$, and $33.3 \%$ for stages I, II, III, and IVA, respectively.

According to Kaplan-Meier 3-year OS analysis and Logrank test in univariate analysis and Cox regression model in multivariate analysis, four factors were statistically significant relation between 3-year OS and early stage $(\mathrm{P}$ $=0.001, \mathrm{OR}=2.52,95 \% \mathrm{CI}=1.34-4.72)$, tumor size $\geq 4 \mathrm{~cm}(\mathrm{P}$ $=0.002, \mathrm{OR}=2.32,95 \% \mathrm{CI}=1.22-4.55)$, pelvic lymph node involvement $(\mathrm{P}=0.002, \mathrm{OR}=2.55,95 \% \mathrm{CI}=1.13-4.48), 1$ week Gap between EBRT and HDR ICRT $(\mathrm{P}=0.002$, OR =
2.04, 95\% CI $=1.29$ - 3.26) and distant metastasis (during the follow up) $(\mathrm{P}=0.001, \mathrm{OR}=8.25,95 \% \mathrm{CI}=3.13-25.2)$, ( $\mathrm{Ta}$ ble 4$)$. Then the favorable prognostic factors in our study in univariate and multivariate analysis were: early stage, tumor size $<4 \mathrm{~cm}$, no pelvic lymph node involvement, 1 week Gap between EBRT and HDR ICRT and no distant metastasis. There was no statistically significant relationship between 3-year OS and histology tumor and mean total dose to the point A by univariate and multivariate analysis (Table 4). 
Rakhsha A et al.

\begin{tabular}{|c|c|c|c|c|c|c|}
\hline Factor & $\begin{array}{c}\text { No. of } \\
\text { Patients }\end{array}$ & $\begin{array}{l}\text { Three- } \\
\text { Year OS }\end{array}$ & $\begin{array}{c}\text { Univariate } \\
\text { Analysis P Value }\end{array}$ & $\begin{array}{c}\text { Odd } \\
\text { Ratio }\end{array}$ & 95\% CI & $\begin{array}{c}\text { Multivariate Analysis } \\
\text { PValue }\end{array}$ \\
\hline Stage & & & 0.02 & 2.52 & $1.34-4.72$ & 0.001 \\
\hline I + IIA + IIB (early stage) & 48 & $38(80.95)$ & & & & \\
\hline III + IVA (locally advanced stage) & 106 & $44(41.50)$ & & & & \\
\hline Histology & & & 0.17 & 1.32 & $0.68-3.49$ & 0.41 \\
\hline Squamous cell carcinoma & 137 & $91(66.42)$ & & & & \\
\hline Adenocarcinoma & 13 & $10(76.92)$ & & & & \\
\hline Adenosquamous carcinoma & 4 & $2(50)$ & & & & \\
\hline Tumor size, $\mathbf{c m}$ & & & 0.02 & 2.32 & $1.22-4.55$ & 0.002 \\
\hline$\geq 4$ & 102 & $41(40.2)$ & & & & \\
\hline$<4$ & 52 & $42(80.76)$ & & & & \\
\hline Pelvic lymph node enlargement & & & 0.02 & 2.55 & $1.13-4.48$ & 0.002 \\
\hline No & 102 & $72(70.58)$ & & & & \\
\hline Yes & 52 & $18(34.6)$ & & & & \\
\hline Distant metastasis (during the follow up) & & & 0.001 & 8.25 & $3.13-25.2$ & 0.001 \\
\hline No & 135 & $85(62.96)$ & & & & \\
\hline Yes & 19 & $2(10.52)$ & & & & \\
\hline Gap between EBRT and HDR ICRT, wk & & & 0.005 & 2.04 & $1.29-3.26$ & 0.002 \\
\hline 1 & 95 & $72(75.78)$ & & & & \\
\hline $3 \geq$ & 59 & $32(54.23)$ & & & & \\
\hline Mean total dose to the point $\mathrm{A}, \mathrm{Gy}$ & & & 0.092 & 1.08 & $0.68-1.64$ & 0.11 \\
\hline$<80$ & 80 & $48(60)$ & & & & \\
\hline$\geq 80$ & 74 & $35(47.3)$ & & & & \\
\hline
\end{tabular}

a Abbreviations: EBRT, external beam radiotherapy; HDR ICRT, high dose rate intracavitary radiotherapy; OS, overall survival.

\section{Discussion}

EBRT with brachytherapy is the standard treatment modality of most stages of carcinoma cervix $(6,7)$. American Brachytherapy Society (ABS) recommends multiple HDR ICRT insertions, allowing tumor volume decreases daily (8). HDR ICRT has been used for more than 40 years in Iran. Our findings were consistent with the most studies that showed similar DFS and OS.

In 2010, Azad and Choudhary (9) showed that 342 patients who had been treated by EBRT and HDR brachytherapy, reported three year disease free survival rate for stages I, II, III, and IV, were $81.8 \%, 70.7 \%, 40.08 \%$, and $11.76 \%$ respectively. The disease free survival rate for early stage (I and II) was 72.04\% and $37.75 \%$ for locally advanced stage (III and IVA).

Likewise in our study, that t 3-year DFS was 85.7\%, $70.7 \%$, $41 \%$ and $16.6 \%$ for stages I, II, III, IVA respectively. The DFS rate was $75 \%$ and $39.62 \%$ for early stage and locally advanced stage $(\mathrm{P}=0.001)$.

Azad and Choudhary (9) also demonstrated that early stage and overall treatment time were prognostic factors that affect the 3 years DFS and 3 years OS with P value of 0.0001 . We also showed that, early stage, tumor size less than $4 \mathrm{~cm}$, no pelvic lymph node involvement and 1 week Gap between EBRT and HDR ICRT were favorable prognostic factors for 3 year DFS by both univariate and multivariate analysis.

Lorvidhaya et al. (10) showed the five-year overall survival rate for Stage IB, IIA, IIB, IIIA, IIIB, IVA, and IVB were $86.3 \%, 81.1 \%, 73.0 \%, 50.3 \%, 47.8 \%, 7.8 \%$, and $30.8 \%$ respective- ly. But they did not report any prognostic factors which affect the 5-year OS.

This finding was partly matched with our study that showed three year OS was $85.7 \%, 76.4 \%, 42 \%$, and $33.3 \%$ for stages I, II, III, and IVA, respectively. In our study, three year OS was $80.95 \%$ for early stage and $41.5 \%$ for locally advanced stage disease $(\mathrm{P}=0.001)$.

But we also showed that, early stage, tumor size $<4 \mathrm{~cm}$, no pelvic lymph node involvement and 1 week Gap between EBRT and HDR ICRT were favorable prognostic factors for 3 year OS by both univariate and multivariate analysis.

Our three year DFS and three year OS were comparable with those in previous reports of brachytherapy in the literature. Factors may affect to this result include high proportional total dose to the point A and physical advantages of HDR brachytherapy compared with LDR and MDR brachytherapy (11-14).

Our study also showed a significant relationship between tumor size (after adjusting for the residual disease after radiation) and pelvic lymph node involvement with the 3 year DFS and 3 year OS with $\mathrm{P}=0.005$ and $\mathrm{P}=0.002$ respectively. This result was comparable with Kodaira and colleagues' findings that demonstrated tumor size and lymph node involvement were prognostic factors. But all patients in that study had stage II cervival cancer (15).

In our study all patients were irradiated with HDR ICRT after EBRT. Therefore, the overall treatment time was increased with the adverse effects. Our study also showed 
a significant relationship between 1 week Gap between EBRT and HDR ICRT versus $3 \geq$ weeks Gap between EBRT and HDR ICRT (overall treatment time $<8$ weeks versus overall treatment time $\geq 10$ weeks) and the 3 year DFS and 3 year $\mathrm{OS}$ with $\mathrm{P}=0.005$ and $\mathrm{P}=0.002$ respectively.

Several similar studies estimated that DFS and OS drops by $1 \%$ per day with prolongation of EBRT and HDR ICRT beyond 8 weeks (16). Then, it should be recommended that to start HDR brachytherapy as short as possible, within 1 week of EBRT.

There was no statistically significant relationship between both 3-year DFS and 3-year OS and histology tumor (after adjusting for the residual disease after radiation) and mean total dose to the point A by univariate and multivariate analysis in our study.

Mosalaei et al. (17) showed overall rectal and bladder complication rates were $34.6 \%$. This finding was consistent with our study that showed similar incidence rate of overall rectal and bladder complication (33.7\%). Overall rectal and bladder complications were seen in 16 (10.4\%) and $36(23.3 \%)$ patients in our study.

There were some limitations in our study. First, our study had a short median follow up duration (38 months). Second, the most of the EBRT was performed by other centers as the best EBRT protocol is usually based at a single institution. However, our study had 154 patients included in the study and relatively few cases (14\%) were excluded.

In conclusion, in this cross sectional-analytic study, early stage, tumor size $<4 \mathrm{~cm}$, no pelvic lymph node involvement and 1 week gap between EBRT and HDR ICRT were independent favorable prognostic factors for three-year DFS. Early stage, tumor size $<4 \mathrm{~cm}$, no pelvic lymph node involvement, 1 week gap between EBRT and HDR ICRT and no distant metastasis (during the follow up) were independent favorable prognostic factors for three-year OS. There was no statistically significant relationship between 3-year DFS, 3-year OS and histology tumor (after adjusting for the residual disease after radiation) and mean total dose to the point A by univariate and multivariate analysis.

Our seven years experience with HDR brachytherapy with Co-60 remote after loading system showed that, HDR brachytherapy in treating patients with carcinoma of cervix was successful after EBRT with acceptable rectal and bladder complications.

\section{Acknowledgements}

We would like to thank the nursing: Ms Khazaie, physic department, technician staff of the radiotherapy department at Shohada-e-Tajrish hospital for their contribution to the treatment and maintenance of our patient records and data collection without whom, carrying out this project would be impossible.

\section{Authors' Contributions}

All authors contributed to this project and article equally. All authors read and approved the final manuscript.

\section{Conflict of Interest}

There is no conflict of interest to be declared.

\section{Financial Disclosure}

None Declared.

\section{References}

1. Jemal A, Bray F, Center MM, Ferlay J, Ward E, Forman D. Global cancer statistics. CA Cancer J Clin. 2011;61(2):69-90.

2. Atahan IL, Onal C, Ozyar E, Yiliz F, Selek U, Kose F. Long-term outcome and prognostic factors in patients with cervical carcinoma: a retrospective study. Int J Gynecol Cancer. 2007;17(4):833-42.

3. Perez CA, Kavanagh BD. Uterine Cervix. In: Halperine EC, Perez CA, Brady LW, editors. Principles and Practice of Radiation Oncology. 5th ed. Philadelphia: Lippincott Williams \& Wilkins; 2008. p. 1532-609.

4. Nagase S, Inoue Y, Umesaki N, Aoki D, Ueda M, Sakamoto H, et al. Evidence-based guidelines for treatment of cervical cancer in Japan: Japan Society of Gynecologic Oncology (JSGO) 2007 edition. Int J Clin Oncol. 2010;15(2):117-24.

5. International Commission on Radiation Units and measurement. Report 38 Dose and volume specification for intracavitary, Inc 1985. USA: Bathesda; 1985. Available from: http://www.icru.org.

6. Orton CG, Seyedsadr M, Somnay A. Comparison of high and low dose rate remote afterloading for cervix cancer and the importance of fractionation. Int J Radiat Oncol Biol Phys. 1991;21(6):1425-34.

7. Orton CG. High Dose Rate Versus Low Dose Rate Brachytherapy for Gynecological Cancer. Semin Radiat Oncol. 1993;3(4):232-9.

8. Nag S, Erickson B, Thomadsen B, Orton C, Demanes JD, Petereit D. The American Brachytherapy Society recommendations for high-dose-rate brachytherapy for carcinoma of the cervix. Int $J$ Radiat Oncol Biol Phys. 2000;48(1):201-11.

9. Azad SK, Choudhary V. Treatment results of radical radiotherapy of carcinoma uterine cervix using external beam radiotherapy and high dose rate intracavitary radiotherapy. J Cancer Res Ther. 2010;6(4):482-6.

10. Lorvidhaya V, Tonusin A, Changwiwit W, Chitapanarux I, Srisomboon J, Wanwilairat S, et al. High-dose-rate afterloading brachytherapy in carcinoma of the cervix: an experience of 1992 patients. Int J Radiat Oncol Biol Phys. 2000;46(5):1185-91.

11. Selke P, Roman TN, Souhami L, Freeman CR, Clark BG, Evans MD, et al. Treatment results of high dose rate brachytherapy in patients with carcinoma of the cervix. Int J Radiat Oncol Biol Phys. 1993;27(4):803-9.

12. Jain VS, Singh KK, Shrivastava R, Saumsundaram KV, Sarje MB, Jain SM. Radical radiotherapy treatment (EBRT + HDR-ICRT) of carcinoma of the uterine cervix: outcome in patients treated at a rural center in India.J Cancer Res Ther. 2007;3(4):211-7.

13. Kagei K, Shirato H, Nishioka T, Kitahara T, Suzuki K, Tomita M, et al. High-dose-rate intracavitary irradiation using linear source arrangement for stage II and III squamous cell carcinoma of the uterine cervix. Radiother Oncol. 1998;47(2):207-13.

14. Chen MS, Lin FJ, Hong CH, Tu CP, Lan JH, Tang SG, et al. High-doserate afterloading technique in the radiation treatment of uterine cervical cancer: 399 cases and 9 years experience in Taiwan. Int J Radiat Oncol Biol Phys. 1991;20(5):915-9.

15. Kodaira T, Fuwa N, Kamata M, Furutani K, Kuzuya K, Ogawa K, et al. Clinical assessment by MRI for patients with stage II cervical carcinoma treated by radiation alone in multicenter analysis: are all patients with stage II disease suitable candidates for chemoradiotherapy? Int J Radiat Oncol Biol Phys. 2002;52(3):627-36.

16. Fyles A, Keane TJ, Barton M, Simm J. The effect of treatment duration in the local control of cervix cancer. Radiother Oncol. 1992;25(4):273-9.

17. Mosalaei A, Mohammadianpanah M, Omidvari S, Ahmadloo N. High-dose rate brachytherapy in the treatment of carcinoma of uterine cervix: twenty-year experience with cobalt after-loading system. Int J Gynecol Cancer. 2006;16(3):1101-5. 